\title{
The N-Glycanase png-1 Acts to Limit Axon Branching during Organ Formation in Caenorhabditis elegans
}

\author{
Nasrin Habibi-Babadi, ${ }^{1}$ Anna Su, ${ }^{1,2}$ Carlos E. de Carvalho, ${ }^{2}$ and Antonio Colavita ${ }^{1,2}$ \\ ${ }^{1}$ Ottawa Health Research Institute, Neuroscience Program and ${ }^{2}$ Heart and Stroke Foundation Centre for Stroke Recovery, University of Ottawa, Ottawa, \\ Ontario K1H 8M5, Canada
}

Peptide: $N$-glycanases (PNGases) are cytoplasmic de- $N$-glycosylation enzymes that have been shown in cultured cells to facilitate the degradation of misfolded glycoproteins during endoplasmic reticulum-associated degradation and in the processing of major histocompatibility complex class I antigens for proper cell-surface presentation. The gene encoding PNGase activity was initially described in budding yeast (Pnglp) and shown to be highly conserved from yeast to humans, but physiological roles in higher organisms have not been elucidated. Here we describe peripheral nervous system defects associated with the first loss-of-function mutations in an animal PNGase. Mutations in png-1, the Caenorhabditis elegans PNGase ortholog, result in an increase in axon branching during morphogenesis of the vulval egg-laying organ and egg-laying behavior changes. Neuronal defects include an increase in the branched morphology of the VC4 and VC5 egg-laying neurons as well as inappropriate branches from axons that run adjacent to the vulva but would normally remain unbranched. We show that png-1 is widely expressed and can act from both neurons and epithelial cells to restrict axon branching. A deletion allele of the DNA repair gene rad-23, orthologs of which are known to physically interact with PNGases in yeast and mammals, displays similar axon branching defects and genetic interactions with png-1. In summary, our analysis reveals a novel developmental role for a PNGase and Rad-23 in the regulation of neuronal branching during organ innervation.

\section{Introduction}

The innervation of peripheral targets involves the remarkable ability of innervating neurons to precisely interpret pathfinding cues along intermediate targets and then respond to specific organ-derived factors that regulate branching to achieve optimal innervation density (Glebova and Ginty, 2005). Morphogenesis of the Caenorhabditis elegans egg-laying system provides an attractive invertebrate model to simplify the task of identifying genes and mechanisms involved in organ formation and innervation.

The nematode egg-laying organ is an anatomically simple structure, consisting of a vulval epithelial channel linking the uterus to the external environment, eight vulval muscles attached to the channel, and the HSN and VC motor neurons that innervate the vulval muscles and coordinate muscle excitations responsible for egg laying (see Fig. 1A) (Li and Chalfie, 1990). Innervation of the vulval muscles occurs in tandem with vulval organogenesis during the fourth larval (L4) stage long after the generation and wiring of sensory and motor circuits and therefore notable as the final neural circuit to form in C. elegans ( $\mathrm{Li}$ and

Received 0ct. 15, 2008; revised 0ct. 20, 2009; accepted Nov. 1, 2009

This work was supported by Canadian Institutes of Health Research Grant MOP-69049. C.E.d.C. received fellowship funding from the Heart and Stroke Foundation Centre for Stroke Recovery. We thank Marc Tessier-Lavigne for his kind help during the initial stage of this project, Oliver Hobert for the ot/s92 reporter, Yuji Kohara for a png-1 CDNA, Shohei Mitani for rad-23(tm2595), Andrea McEwan for technical assistance, and the Caenorhabditis Genetics Center and the C. elegans Gene Knockout Consortium for providing strains. We also thank Claudia Arauz, Tadash Suzuki, and lan Chin-Sang for critical reading of the manuscript.

Correspondence should be addressed to Antonio Colavita, Ottawa Health Research Institute, Neuroscience Program, 451 Smyth Road, University of Ottawa, Ottawa, Ontario K1H 8M5, Canada. E-mail: colavita@uottawa.ca. DOI:10.1523/JNEUROSCI.4962-08.2010

Copyright $\odot 2010$ the authors $\quad 0270-6474 / 10 / 301766-11 \$ 15.00 / 0$
Chalfie, 1990; Garriga et al., 1993). Cell ablation and genetic studies have shown that vulval epithelial cells play an essential role in the innervation and shaping of the egg-laying circuit by providing a source of axon guidance, axon branching, and synapse formation cues (Li and Chalfie, 1990; Garriga et al., 1993; Colavita and Tessier-Lavigne, 2003; Shen et al., 2004; Adler et al., 2006).

Here we describe a novel function for PNG-1, a peptide: $N$ glycanase (PNGase), in limiting the amount of neuronal branching during innervation of the vulval egg-laying organ in $C$. elegans. PNGase is a highly conserved cytoplasmic enzyme that is solely responsible for the de- $N$-glycosylation of $N$-linked glycoproteins and glycopeptides in the cytosol of eukaryotic cells (Suzuki, 2007). PNGases cleave $N$-linked glycoamide bonds in a reaction that releases a free oligosaccharide and converts the hydrolyzed asparagine residue to aspartate (Suzuki, 2007). A role for PNGase in $\mathrm{N}$-glycoprotein catabolism was revealed with the elucidation of the endoplasmic reticulum (ER)-associated degradation (ERAD) pathway, an ER surveillance system that actively recognizes and retrotranslocates misfolded or aberrant proteins from the ER to the cytosol for proteasomal degradation. ERADtargeted glycoproteins are catabolized in the cytosol by a combination of PNGase-mediated deglycosylation and degradation by the proteasome (Hirsch et al., 2003; Blom et al., 2004; Kim et al., 2006). In mammalian cells, PNGases have also been implicated in the cytoplasmic processing of $N$-glycosylated peptide fragments into epitopes that maximize major histocompatibility complex (MHC) class I cell-surface antigenicity (Selby et al., 1999; AltrichVanLith et al., 2006; Kario et al., 2008).

Despite these recent findings, the physiological roles for cytosolic $N$-glycanases remain unknown. Phenotypic analysis of 
A

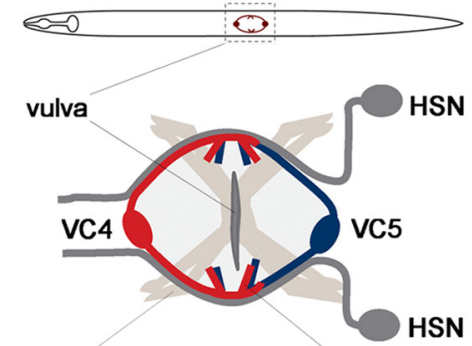

vulval muscles
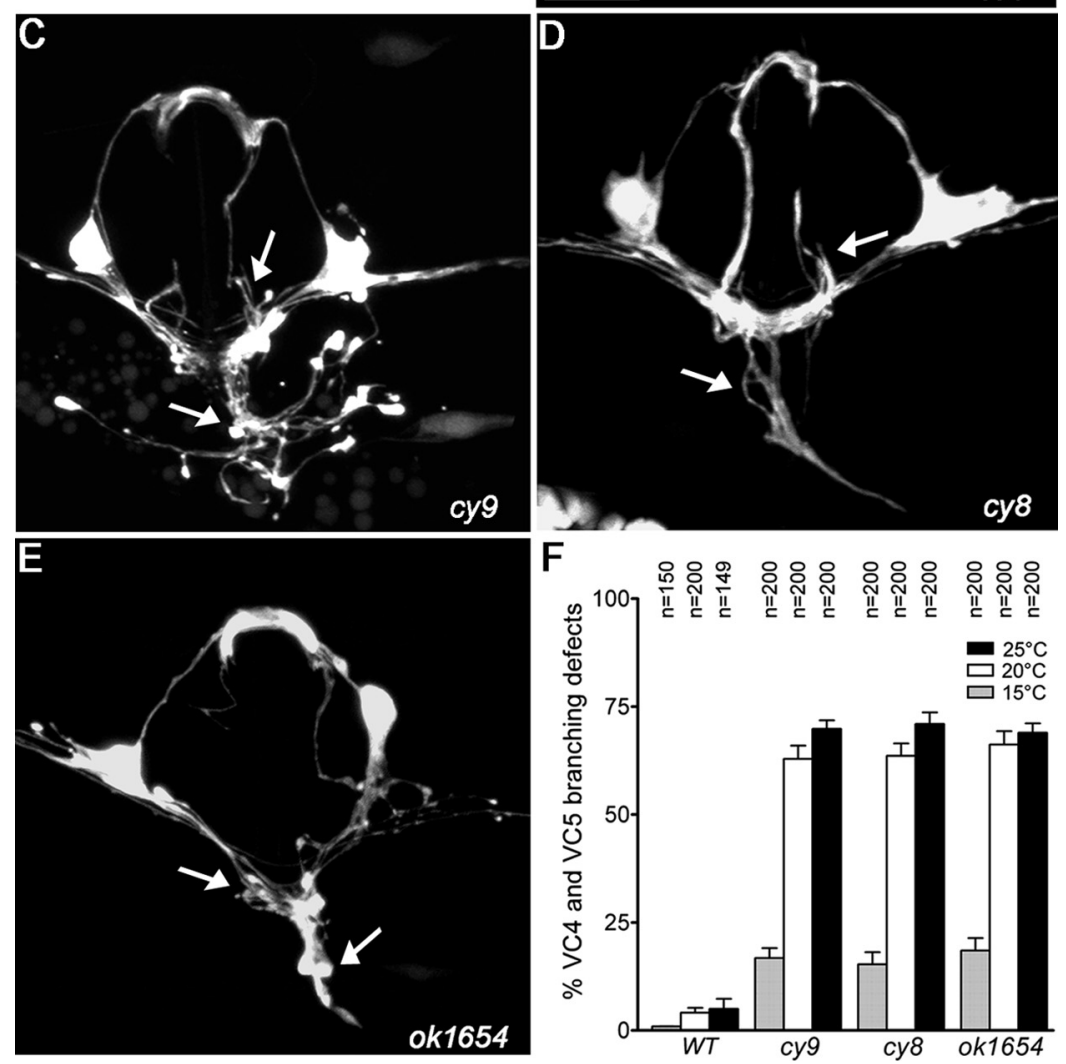

Figure 1. Mutants displaying excessively branched VC4 and VC5 axons. $A$, Schematic of a worm (ventral view) showing the positions of VC4 and VC5 and the vulval egg-laying organ innervated by the HSN and VC motor neurons. $\boldsymbol{B}-\boldsymbol{E}$, Ventral view of VC4 and VC5 neurons in adult wild-type $(\boldsymbol{B} ; \mathrm{WT})$ and $c y 9$, cy8, and ok1654 (C-E) mutants visualized using a cy/s4[Pcat-1::GFP] reporter. Arrows indicate axon branching defects. $\boldsymbol{F}$, Quantification of VC4-5 excess branching defects in wild-type and mutant animals. Branching defects decrease when animals are grown at lower temperature $\left(15^{\circ} \mathrm{C}\right)$. Error bars denote SEM; $n=150-200$ animals. Scale bar, $10 \mu \mathrm{m}$.

PNGase mutants in the budding yeast Saccharomyces cerevisiae (Suzuki et al., 2000) and the plant Arabidopsis thaliana (Diepold et al., 2007), which could have shed light on in vivo roles, were not overly informative because mutants lacked obvious abnormalities. A PNGase mutant in Neurospora crassa, a filamentous fungus, displays a noticeable hyphal growth defect, the underlying cause of which is not known (Seiler and Plamann, 2003). In this study, we report our phenotypic analysis of the first loss-offunction mutations in an animal PNGase revealing a novel developmental role for this highly conserved enzyme in neuronal branching during organ innervation.

\section{Materials and Methods}

Genetics and mapping. C. elegans strains were grown on nematode growth medium using standard methods. In addition to png-1 (cy8, cy9, ok1654), the following mutations were used: bli-4(e937), egl-1(n986), hrd-1(tm1543), ire-1(ok799), pek1(ok275), rad-23(tm2595), unc-30(e191), and unc-75(e950). The following strains were used: cyIs4[Pcat-1::GFP] to label VC4 and VC5, kyIs179[Punc-86::GFP] to label HSN, unc30(e191); juIs76[Punc-25::GFP] to label AVL and DVB, and otIs92[Pflp-10::GFP] to label DVB neurons. $c y 9$ was mapped using standard N2/CB4856 single nucleotide polymorphism (SNP) mapping methods within the bli-4 unc-75 interval on linkage group I (LGI) by isolating $51 \mathrm{Bli}$ non-Unc progeny from a bli4(e937) cy9 unc-75(e950)/Hawaiian (N2/ CB4856) parent in which 43 recombinants were $c y 9 /+$ and eight were $c y 9 / c y 9$. cy9 was then mapped more precisely to a small interval between cosmids W02B9 and ZK1025 using SNP markers within the bli-4 unc-75 interval. png-1 and $\mathrm{rad}-23$ alleles were backcrossed at least three times. Double mutants were constructed using standard methods and confirmed by complementation test or PCR genotyping (primer sequences available on request).

Molecular biology and transgenic animals. png-1/F56G4.5 was cloned by examining transgenic animals injected with cosmid pools, genomic PCR fragments, or subclones (at 10 $\mathrm{ng} / \mu \mathrm{l}$ each along with $25 \mathrm{ng} / \mu \mathrm{l}$ Podr-1::RFP coinjection marker) within the genetically mapped interval for rescue of $c y 9$ branching defects. Mutations in $c y 8$ and $c y 9$ were identified by sequencing PCR amplified exons from genomic DNA. rad-23 defects were rescued with a $3 \mathrm{~kb}$ genomic PCR fragment containing $1.5 \mathrm{~kb}$ upstream and $0.35 \mathrm{~kb}$ downstream of the start and stop, respectively. The transcriptional Ppng-1::GFP (pAC3) reporter was made by PCR amplifying $\sim 600$ bp upstream of the predicted F56G4.4-F56G4.5 operon start site and cloning upstream of green fluorescent protein (GFP) in pPD95.77 (Fire Lab Vector kit). The translational Ppng-1::PNG-1::GFP (pAC7) fusion was made by inserting the open reading frame from a cDNA ( $y k 811 \mathrm{~b} 02$; a gift from Y. Kohara, Genome Biology, National Institutes of Genetics, Mishima, Japan), minus stop codon, in frame between the promoter and GFP in pAC3. Expression clones for cell-specific studies were made by replacing the png-1 promoter in pAC7 with a $2.4 \mathrm{~kb}$ cat-1 (Colavita and Tessier-Lavigne, 2003), $2.6 \mathrm{~kb}$ unc-4 (Lickteig et al., 2001), 1.2 $\mathrm{kb}$ col-10 (Colavita and Tessier-Lavigne, 2003), $0.9 \mathrm{~kb}$ ajm-1 (Köppen et al., 2001), $1.5 \mathrm{~kb}$ rgef-1 (AltunGultekin et al., 2001), $3 \mathrm{~kb}$ egl-17 (Cui and Han, 2003), $1.3 \mathrm{~kb}$ sqv-1, $0.9 \mathrm{~kb} s q v-3$ (Hwang and Horvitz, 2002), or $0.4 \mathrm{~kb}$ ocr-4 (Jose et al. 2007) promoter. A PCR-based deletion strategy using pAC7 as a template was used to generate constructs containing in-frame deletions of the thioredoxin (residues 2-100) or C-terminal (residues 426-600) domain. Site-directed mutagenesis (QuickChange XL; Stratagene) was used to convert the two active site cysteines in the thioredoxin domain (pAC7) to two serines. Cell-specific rescue and structure-function analysis was performed by testing for rescue of VC branching defects in png-1(cy9); cyIs4 animals after germ-line transformation. Plasmids were injected at $20-25 \mathrm{ng} / \mu \mathrm{l}$ along with Podr-1::RFP at $45 \mathrm{ng} / \mu \mathrm{l}$ except for the rad-23 genomic fragment that was injected at $1 \mathrm{ng} / \mu \mathrm{l}$. Transgenic lines were maintained by following red fluorescent protein (RFP) fluorescence. PCR primer sequences are available on request. 
Characterization of neuronal phenotypes. In late L4 and young adults, VC4 and VC5 axons partially overlap to form a circular tract around the vulva with distinct left and right branching patterns. VC neurons were scored as excessively branched at the young adult stage if more than 10 branches were observed on either the left or right side compared with the three to five branches normally observed in wild type. Because VC4 and VC5 axons fasciculate at the site of branching, individual branches could not be unambiguously identified, and therefore VC4 and VC5 axon branches were pooled for scoring purposes. AVL and DVB axons were scored as displaying an axon branch if a distinct axonal protrusion at least $3-5 \mu \mathrm{m}$ in length (approximately one neuronal cell body diameter) was observed. DVB axons normally terminate a short distance posterior to the vulva. A termination defect was scored as any DVB process that extends dorsally along the vulval epithelium or anteriorly past the vulva. Animals were mounted on 2\% agarose pads in M9 buffer containing $5 \mathrm{~mm}$ levamisole and examined by epifluorescence on a Zeiss Axioplan2. Images were captured using a Zeiss LSM510 confocal microscope.

Egg-laying assays. Egg-laying and eggretention assays were performed as described previously (McGovern et al., 2007). Briefly, synchronized mid-L4 stage larvae were allowed to grow to young adults containing a single row of eggs in utero. Individual animals were then transferred to food plates for $2 \mathrm{~h}$ at $20^{\circ} \mathrm{C}$, after which the animal was removed and the number of eggs was counted. The same plates were scored again after $48 \mathrm{~h}$ to confirm progeny count. For egg-retention assays, synchronized late-L4 larvae were allowed to grow on plates with bacteria at $20^{\circ} \mathrm{C}$. At $24 \mathrm{~h}$ later, young adults were placed on glass slides, and the number of eggs in utero was counted under a $40 \times$ objective.

\section{Results}

\section{A PNGase (png-1) is disrupted in VC} axon branching mutants

The $c y 8$ and $c y 9$ alleles were identified in a genetic screen for VC4 and VC5 (VC4-5)

branching abnormal mutants using a cyIs4[Pcat-1::GFP] reporter to visualize the VC4-5 egg-laying neurons (A. Colavita and Marc Tessier-Lavigne, unpublished observations). These mutants display a highly penetrant increase in the complexity of the VC4-5 branching morphology compared with wild type (Fig. $1 B-D$ ).

An SNP-based mapping strategy was used to map cy9 to a small interval on LGI between cosmids W02B9 and ZK1025. To identify the gene disrupted in our mutants, we generated transgenic lines carrying PCR amplified genomic or cosmid DNA located within this interval and scored for rescue of the VC4-5 branching defects (Fig. $2 A)$. A genomic fragment containing the cotranscribed genes F56G4.4 and F56G4.5 but not deletion derivatives that removed F56G4.5 rescued the branching phenotype. F56G4.5 was verified as the $c y 9$ locus by obtaining rescue with a full-length cDNA driven by $\sim 600$ bp of upstream sequence, identifying the DNA lesions in cy8 and cy9, and showing that ok1654, an F56G4.5 deletion allele obtained from the C. elegans Gene Knockout Consortium, displays similar defects.

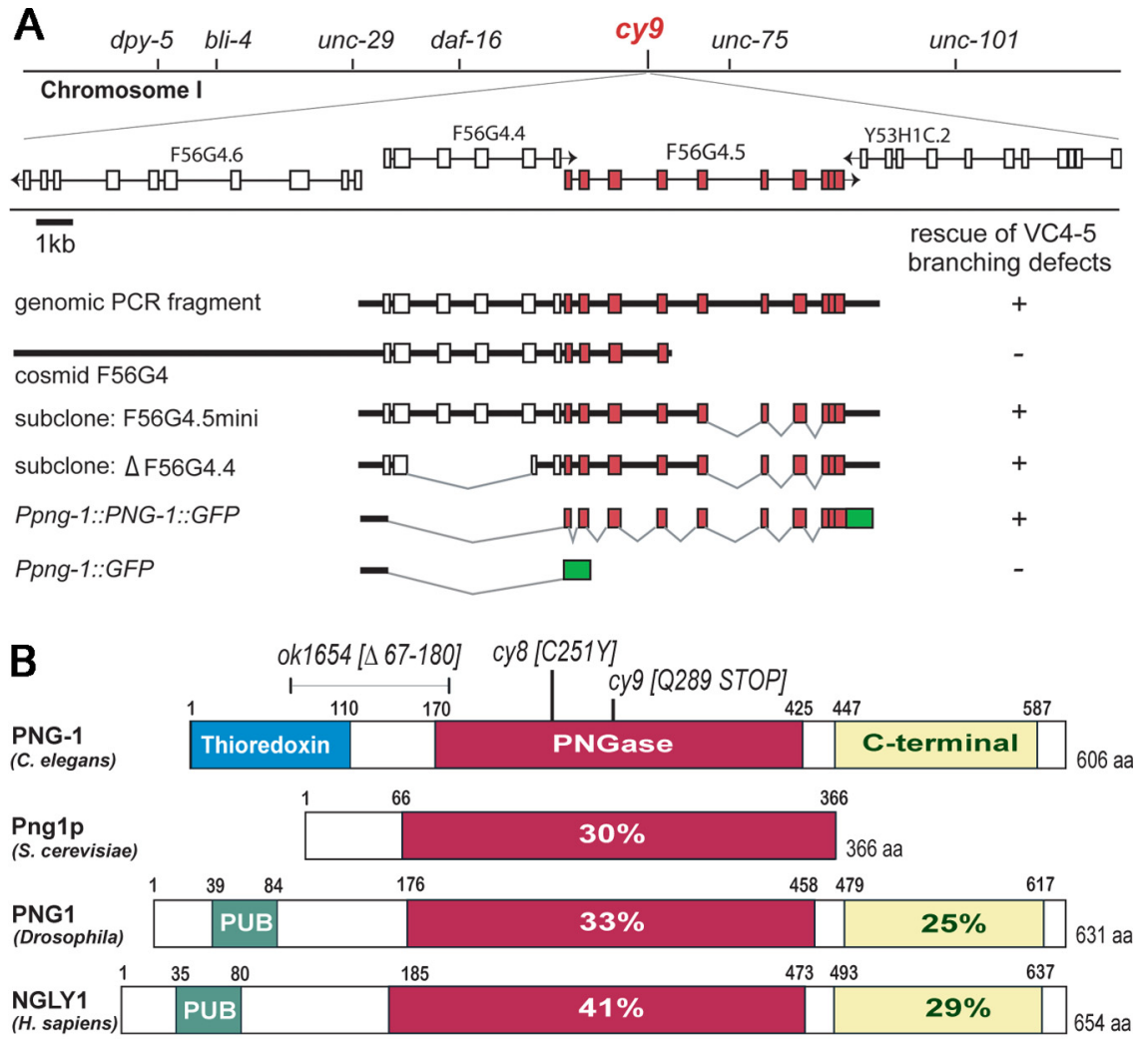

C

CePNG-1 245 QTRTGRCGEWANCFGLLLAALNLESRFIYDTTDHVWNEVYLLAEQRWCHVDPCEN 299

ScPng1 183 ETRKGRCGEWCNLFTLILKSFGLDVRYVWNREDHVWCEYFSNFLNRWVHVDSCEQ 237

88 VSRKGRCGEYANCFTFLCRAIDYDARIVHSHFDHVWTEVYSEAQMRWLHVDPSEN 343

DINglY1 287 ETRKGRCGEWANCFTLLCRALGLEARYIWDSTDHVWTEVYSQSQRRWIHCDPCEN 342

hNgly1 302 ETRCGRCGEWANCFTLCCRAVGFEARYVWDYTDHVWTEVYSPSQQRWLHCDACED 357

$*$

Figure 2. VC axon branching mutants disrupt the PNGase ( $p n g-1)$ gene. $\boldsymbol{A}$, The $c y 9$ mutant was mapped to a small genetic nents and a png-1 translational fusion to GFP (green). B, Domain organization of PNG-1 and orthologs showing percentage domains. Molecular lesions in png-1 are indicated in $B$ and $C$, and asterisks mark the invariant Cys, His, Asp active site triad. $C$, $C$ elegans (GenBank accession number AAF74721); Sc, S. cerevisiae (GenBank accession number S61970); Dm, Drosophila (GenBank accession number AAF74722); Dr, zebrafish (GenBank accession number AAH95313); m, mouse (GenBank accession number AAF74723); h, human (GenBank accession number AAF74720). ClustalW alignment (version 6.0).

F56G4.5 encodes the PNGase PNG-1 (Fig. 2B), a 606 amino acid protein consisting of an $\mathrm{N}$-terminal thioredoxin domain, a central PNGase domain, and a predicted C-terminal mannose binding domain (Suzuki et al., 2007). PNGases belong to the transglutaminase (TGase) superfamily characterized by a highly conserved catalytic active site consisting of Cys, His, and Asp residues (Cys251, His278, and Asp295 in PNG-1) that are essential for $N$-glycanase activity (Fig. 2C). Animal PNGases differ from those of lower eukaryotes in having extended $\mathrm{N}$ - and C-terminal sequences. Mammalian and fly PNGases contain an N-terminal PUB domain known to interact directly with components of the ubiquitinproteasome pathway and a C-terminal mannose binding domain (Suzuki, 2007). With the exception of a divergent N-terminal thioredoxin domain, which so far appears to be found only in some nematode PNGases, PNG-1 is a clear ortholog of S. cerevisiae Pnglp and human NGly1 displaying 30 and 41\% identify in the PNGase domain, respectively (Fig. 2B) (Suzuki et al., 2000, 2007). 
The molecular lesions in png-1 alleles were identified (Fig. $2 B, C) . c y 9$ is a candidate null allele because it introduces a stop at Q289 that should result in a premature termination within the PNGase domain and therefore abolish PNGase activity. cy8 is a missense mutation that substitutes the invariant C251 catalytic residue in the PNGase active site with a tyrosine. ok1654 is a 1.1 $\mathrm{kbp}$ deletion predicted to generate an in-frame deletion of residues 67-180.

\section{png-1 mutants display an increase in VC neuronal branching} at the vulva

VC4-5 neurons are born during late L1 (first larval stage) but delay axon outgrowth until early L4 after the onset of vulval organogenesis (Li and Chalfie, 1990). During L4, VC4-5 axons project laterally around the developing vulva, eventually overlapping and forming branches or arborizations at distinct sites on the left and right sides of the vulva where connections are made with presynaptic (HSN) and postsynaptic (vulval muscles) partners (Shen et al., 2004; Feinberg et al., 2008). In png-1 mutants, VC4-5 neurons display more highly branched terminal arbor morphologies characterized by an increase in branch number and branch length (Fig. $1 B-E$ ). The phenotype is highly penetrant with 70,71 , and $69 \%$ of $c y 9, c y 8$, and $o k 1654$ animals displaying excess VC branching morphologies at $25^{\circ} \mathrm{C}$, respectively (Fig. $1 F$ ). We note that VC mutant defects are highly variable with supernumerary branches extending in multiple directions along the vulval or body wall epithelium either in isolation or as loosely fasciculated bundles that often intersected. This morphology was further complicated by the normal overlap of the VC4-5 arbors that together precluded the unambiguous scoring of branch number and branch length. Defects appear specific to axon branching because VC axon outgrowth around the vulva was unaffected, suggesting that growth cone motility was not impaired. We also did not observe any other obvious morphological, locomotion, or viability defects in mutant animals.

The excessive VC branching phenotype is temperature sensitive and primarily ameliorated when animals are grown at $15^{\circ} \mathrm{C}$ instead of $20^{\circ} \mathrm{C}$ or $25^{\circ} \mathrm{C}$ (Fig. $1 \mathrm{~F}$ ). Because all the molecular lesions appear to be null or strong loss-of-function mutations, this phenotype is not consistent with allele-specific effects on protein stability. Such temperature-dependent mitigation of neuronal morphology defects have been observed by others in C. elegans and are likely the result of the action of an underlying temperaturesensitive process (Peckol et al., 1999).

\section{png-1 mutants display egg-laying behavior defects}

The VC4-5 morphology defects in png-1 mutants suggested a possible impact on egg-laying behavior. HSN and VC4-5 motor neurons innervate the vulval muscles to stimulate egg laying. Studies in which these neurons have been selectively ablated show that loss of VC4-5 result in less severe egg-retention defects compared with loss of the HSNs, but loss of both abolish egg laying (Waggoner et al., 1998; Bany et al., 2003). In addition to their role in stimulating egg laying, VC4-5 neurons also inhibit egg laying through modulation of HSN activity (Bany et al., 2003). An examination of HSN neurons using a Punc-86::GFP reporter did not reveal branching or morphology defects in mutant animals (supplemental Table 1, available at www.jneurosci.org as supplemental material).

To determine whether increased VC arborization correlates with changes in egg-laying behavior, we examined png-1 mutants for egg-laying phenotypes. In a measure of the rate of egg laying in synchronized individual young adults carrying a single row of
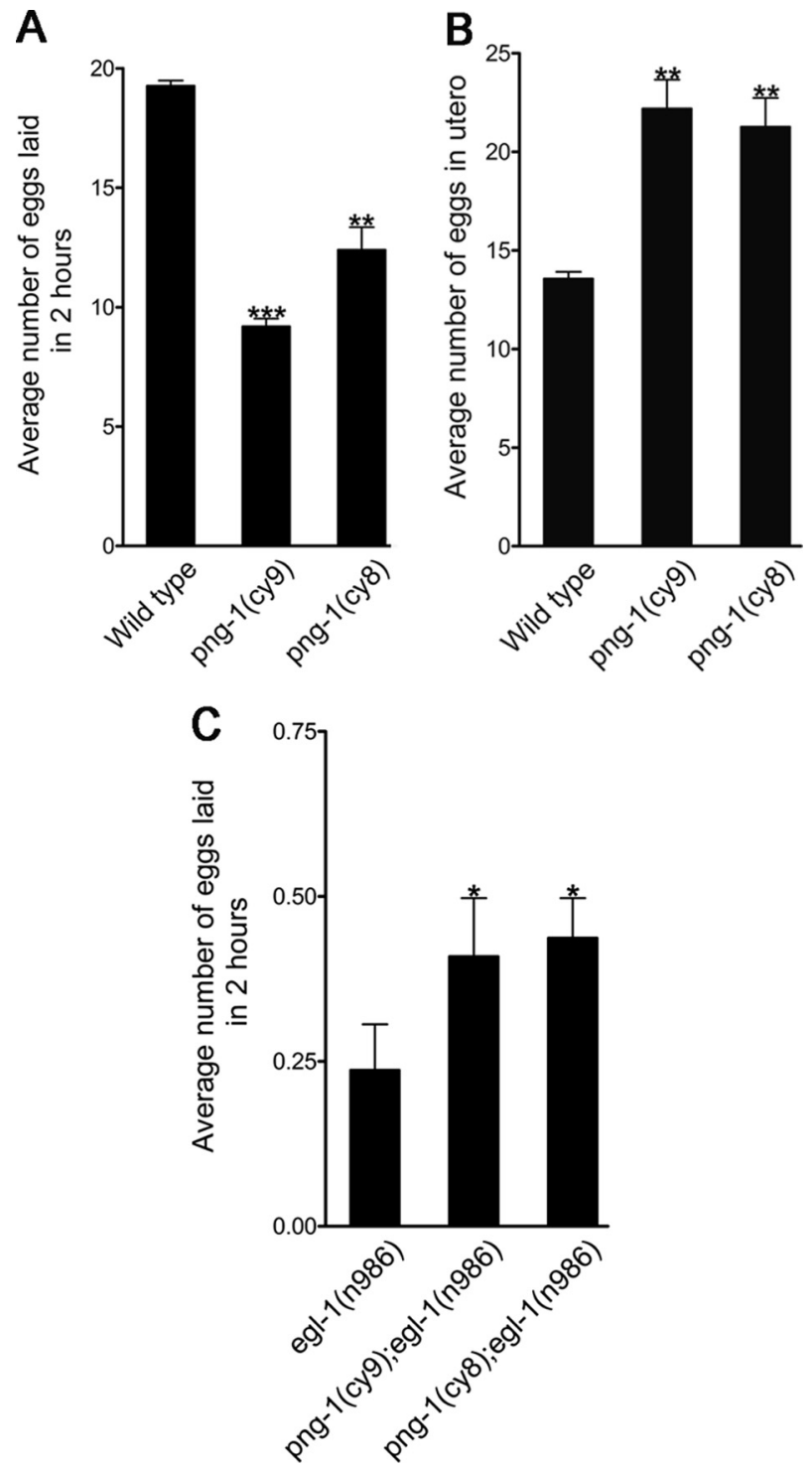

Figure 3. png-1 is involved in egg-laying behavior. $\boldsymbol{A}, \boldsymbol{B}$, png-1 mutants have a mild Egl phenotype characterized by a reduced rate of laid eggs $(\boldsymbol{A})$ and in utero retention of developing embryos (B). C, png-1 deficiency partially suppresses the egg-retention phenotype of an egl-1 gain-of-function mutant. HSN neurons undergo cell death in egl-1(n986) resulting in a genetic background in which egg-laying behavior is completely dependent on VC innervation of the vulval muscles. Error bars denote SEM. ${ }^{*} p<0.05,{ }^{* *} p<0.001,{ }^{* * *} p<0.0001, t$ test.

eggs in utero, we found that png-1 mutants displayed a decrease in the eggs laid per $2 \mathrm{~h}$ period ( 12.9 for $c y 9$ and 9.0 for $c y 8)$ compared with wild-type controls (19.4) (Fig. 3A). We also found an eggretention phenotype in mutants (22.2 for $c y 9$ and 21.3 for $c y 8$ ) when counting the number of eggs in the uterus of synchronized young adults compared with wild-type controls (13.8) (Fig. 3B). These findings suggest that VC morphology defects in png-1 mutants may interfere with normal egg-laying behavior.

In a third assay, we took advantage of an egl-1(n986) gain-offunction mutant in which HSN neurons undergo cell death to create a background in which egg laying is solely dependent on VC neuronal function (Waggoner et al., 1998). egl-1(n986) animals display a severe egg-laying retention phenotype. In the assay of the number of eggs laid in a $2 \mathrm{~h}$ period, we found that $90 \%$ of egl-1(n986) animals $(n=300)$ failed to lay any eggs, resulting in an average of 0.19 eggs laid per $2 \mathrm{~h}$ period (Fig. $3 C$ ). We hypothesized that, if our VC morphology defects interfered with VC 
neuronal activity or vulval muscle innervation, we would expect to see an enhancement of the egg-laying defects in an egl-1(n986) background. Instead, we found that png-1(cy9); egl-1(n986) and png-1 (cy8); egl-1 (n986) animals displayed a significant increase in the average number of eggs laid per $2 \mathrm{~h}$ period $(0.45$ and 0.47 eggs respectively; $n=300-330$ ) compared with egl-1 single mutants. This is consistent with increased VC branching in png- $1^{-/-}$animals, causing increased stimulation of vulval muscles and partially compensating for the loss of the egg-laying stimulatory effect of HSN innervation.

\section{png-1 mutants display an increase}

in axonal branching along the

\section{vulval epithelium}

To determine the scope and nature of the neuronal defects in png-1 mutants, we used GFP reporter transgenes to label subsets of anteroposterior and dorsoventral projecting axon tracts (supplemental Table 1, available at www.jneurosci.org as supplemental material). Axon pathfinding and morphology along the major body axes was found to be normal in png- 1 animals except for the occurrence of inappropriate branches in a subset of ventral cord axons visualized with the juIs76[Punc-25::GFP] reporter at sites that correlated precisely with the position of the vulva.

Punc-25::GFP labels all GABAergic neurons, only a subset of which possess processes in the ventral nerve cord that run adjacent to the vulva $(\mathrm{DD}, \mathrm{VD}, \mathrm{AVL}$, and DVB). We took advantage of the fact that $u n c-25$ promoter activity in the DD and VD neurons is silenced in an unc$30^{-1-}$ background to visualize the AVL neuron (Eastman et al., 1999). We found that AVL, which normally projects an unbranched axon from a cell body located in the head to its target in the tail, displays inappropriate branches at the vulva in png-1 mutants ( $25 \%$ in $c y 9 ; n=150$ ) (Fig. $4 A-D)$. The DVB neuron, visualized with an otIs92[Pflp-10::GFP] reporter (Mehta et al., 2004), which normally projects an axon from a cell body in the tail to a region just posterior to the vulva, displays an axon guidance defect in which axons overextend dorsally along the vulval epithelium or anteriorly past the vulva (36\% in cy9; $n=149$ ) (Fig. $4 E-G, I)$. A subset of the DVB axons that overextend and reach the vulva also show inappropriate branching along the vulval epithelium $(10 \%$ in $c y 9 ; n=87)$ (Fig. $4 H, J)$. AVL and DVB branching phenotypes were not observed at the L3 stage before vulval organogenesis (Fig. $4 D, I J)$ ). This is consistent with previous findings that vulval cells are a source of a branch promoting activity (Li and Chalfie, 1990). Interestingly, an in examination of DVB morphology in a png-1 $1^{-1-}$, vulvaless mutant background revealed a suppression of
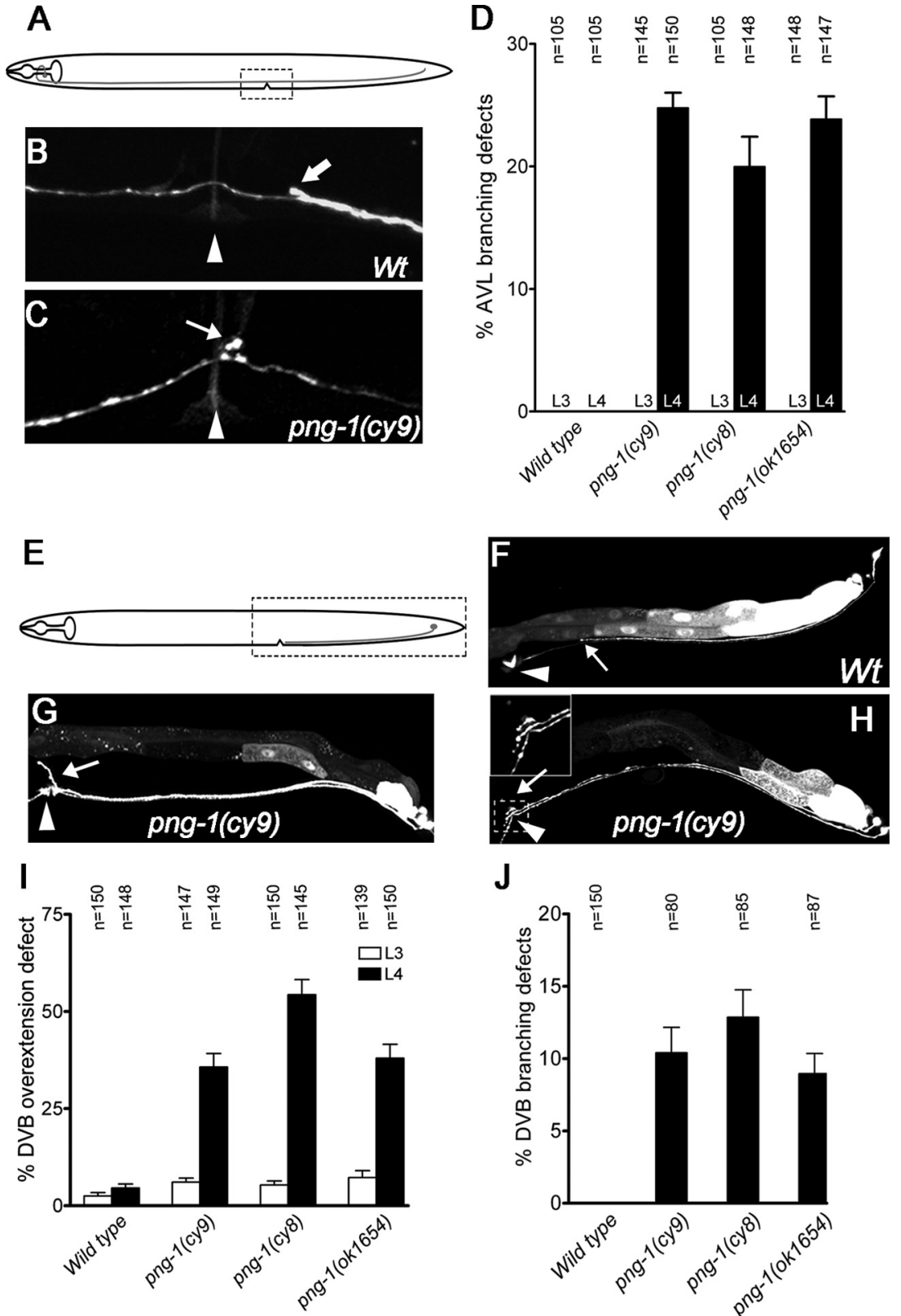

$\mathrm{J}$

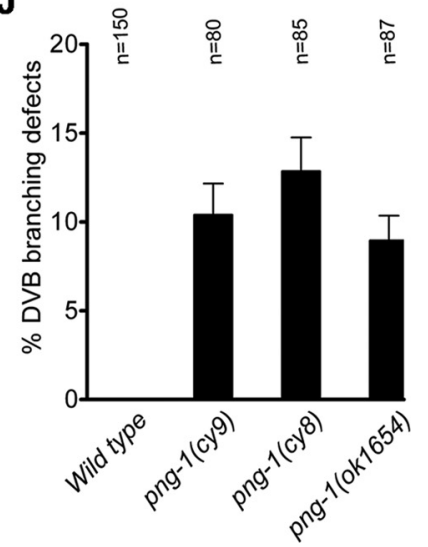

Figure 4. Several neurons display excessive or inappropriate axonal branching at the vulva in $p n g-1$ mutants. $A, E$, Schematics of AVL and DVB neurons with imaged areas boxed. B, C, An AVL axon, visualized in a Punc-25::GFP; unc-30(e191) background, running adjacent to the vulva (arrowhead) in a wild type $(\boldsymbol{B} ; \mathrm{Wt})$ and displaying an inappropriate branch (arrow) in a png-1(cy9) adult ( $\boldsymbol{C}$. Thick arrow (B) points to a DVB axon. $\boldsymbol{D}$, Inappropriate AVL branches are observed at mid-to-late $L 4$ stage at the vulva but not before vulval organogenesis at the $L 3$ stage in png-1 mutants. $\boldsymbol{F}-\boldsymbol{H}, \mathrm{A}$ DVB axon visualized using an Pflp-10::GFP reporter terminating (arrow) posterior to the vulva (arrowhead) in a wild type $(\boldsymbol{F})$, overextending anteriorly and dorsally along the vulval epithelium (arrow) $(\boldsymbol{G})$ and displaying a vulval branch (arrow) in png-1(cy9) adults $(\boldsymbol{H})$. Pflp-10::GFP is also variably expressed in the VulD vulval cells (arrowhead in $\boldsymbol{H}$ ). I, J, DVB axon overextension defects are more severe at the mid-to-late L4 stage compared with the $L 3$ stage in png-1 mutants $(\boldsymbol{I})$. A subset of DVB axons that display overextension defects also branch at the vulva $(\boldsymbol{J})$. Error bars denote SEM. Scale bars: $\boldsymbol{B}, \boldsymbol{C}, 10 \mu \mathrm{m} ; \boldsymbol{F}-\boldsymbol{H}, 20 \mu \mathrm{m}$.

ectopic DVB vulval branches as expected but not DVB overextension (data not shown), suggesting that these phenotypes are separable and that, although vulval cells are involved in DVB branching, they may not contribute to DVB axon termination. Overall, these results indicate that png-1 acts to limit the extent of axon branching and to prevent subsets of axons from branching inappropriately at the vulva.

\section{png- 1 is widely expressed}

As a first step in characterizing png- 1 expression, we made a Ppng-1::GFP transcriptional reporter using the $\sim 600$ bp pro- 


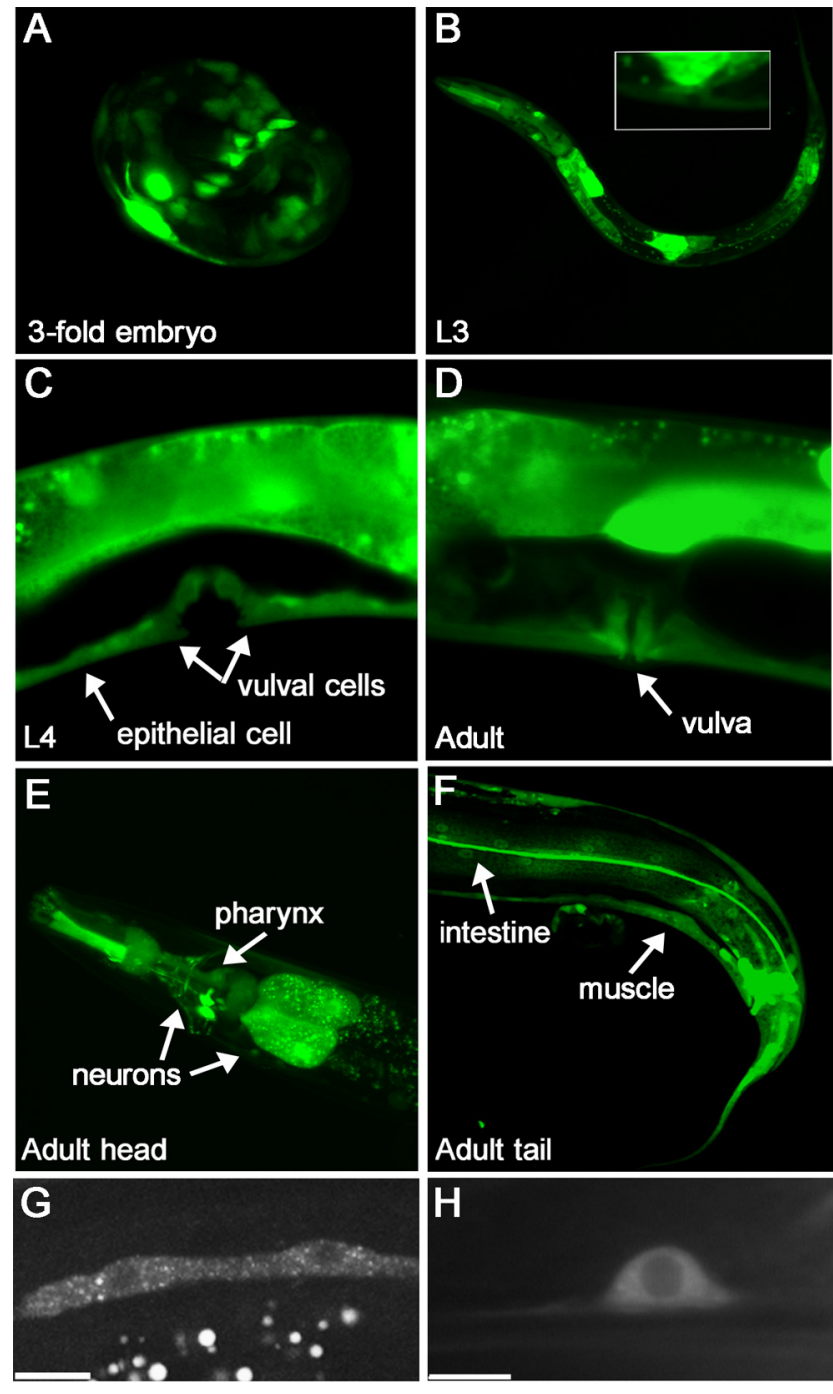

Figure 5. $\quad p n g-1$ is widely expressed. $\boldsymbol{A}-\boldsymbol{F}, p n g-1$ promoter activity at various developmental stages visualized using a Ppng-1::GFP transcriptional reporter (see Fig. 2A). The $\sim 600$ bp promoter region was shown to be sufficient to rescue $\mathrm{VC}$ branching defects in png- 1 mutants. Promoter activity was found in multiple tissues, including epithelium, neurons, muscle, intestine, and vulval epithelial cells. Inset in $\boldsymbol{B}$ shows magnification of $L 3$ vulval region. $\boldsymbol{G}, \boldsymbol{H}$, PNG-1::GFP expressed from a pan-epithelial or pan-neuronal promoter shows cytoplasmic localization. Epithelial seam cells $(\boldsymbol{G})$ and a tail neuron $(\boldsymbol{H})$ are shown. Scale bars, $10 \mu \mathrm{m}$.

moter that was shown to be sufficient in png- 1 rescue experiments. Transgenic animals carrying this reporter showed broad png-1 transcriptional activity during all developmental stages in most tissues, including neurons, gonad, pharynx, intestine, body wall muscle, and epithelial cells including vulval cells (Fig. 5). Widespread expression has also been reported for plant and mammalian PNGases (Kitajima et al., 1995; Diepold et al., 2007), as might be expected for a housekeeping gene involved in glycoprotein catabolism. In the egg-laying system, promoter activity was detected consistently in all vulval cells from the late L3 stage to adulthood (Fig. 5B-D).

To determine subcellular localization, we generated Ppng-1:: $P N G-1:: G F P$, a translational GFP fusion. The expression and functionality of this construct was confirmed by transgenic rescue of the VC axon branching defects in png-1 animals (Fig. 2 B). However, we were unable to detect GFP fluorescence in animals carrying this transgene either directly or by indirect immunofluorescence using anti-GFP antibodies (data not shown). To ad-

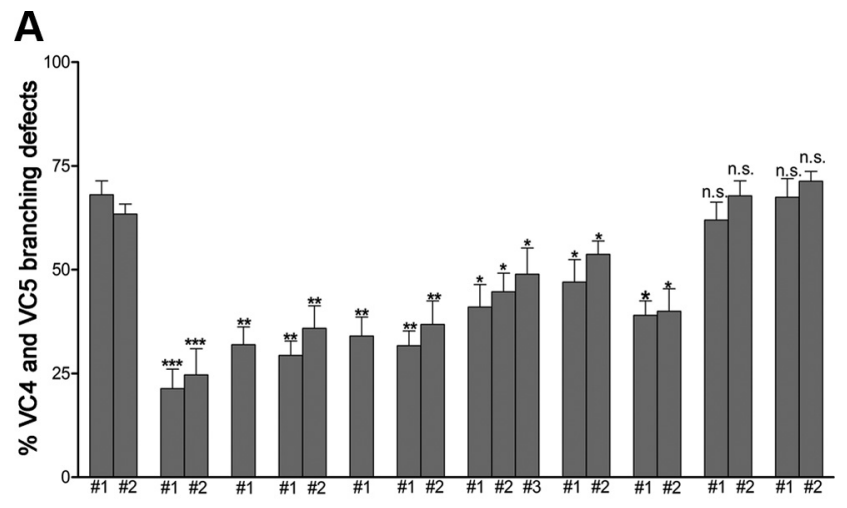

Vector Ppng-1 Pajm-1 Pcol-10 Psqv-1 Psqv-3 Pcat-1 Punc-4 Prgef-1 Pegl-17 Pocr-4
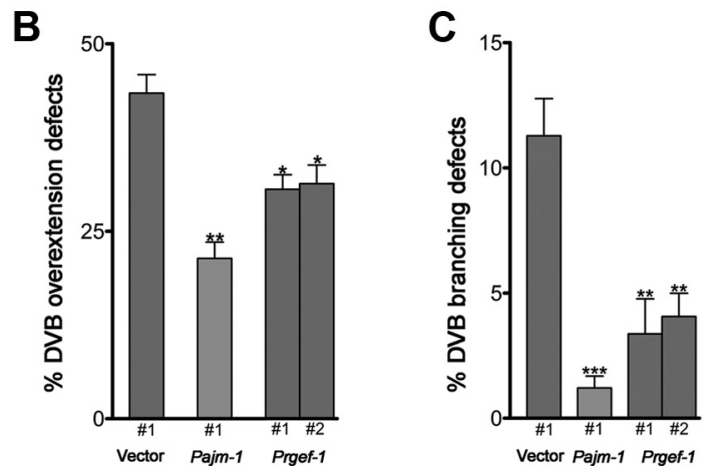

Figure 6. png-1 can act from epithelial cells or neurons to regulate VC axon branching. $\boldsymbol{A}$, Cell-specific promoters were used to drive png-1 expression in epithelial cells or neurons. Constructs were injected along with odr-1::RFP injection marker into png-1(cy9); cy/s4 [cat-1p::GFP] animals to generate multiple independent extrachromosomal transgenic lines. Lines expressing png- 1 from pan-epithelial (col-10, ajm-1) or pan-neuronal (rgef-1) promoters rescue VC4-5 branching defects. png-1 expression in vulval cells (sqv1, sqv-3) or VC4-5 (cat-1) is sufficient for rescue. $\boldsymbol{B}, \boldsymbol{C}$, DVB axon overextension $(\boldsymbol{B})$ and branching $(\boldsymbol{C})$ defects are rescued with both pan-epithelial (ajm-1) and pan-neuronal (rgef-1) expressed png-1. Controls are empty vectors. Error bars denote SE of proportion; $n=62-200$ animals. ${ }^{*} p<0.05,{ }^{* *} p<0.01$, ${ }^{* * *} p<0.001, t$ test.

dress the possibility that broad overexpression of PNG-1 from its own promoter may be detrimental, we drove PNG-1::GFP from pan-epithelial (col-10) or pan-neuronal (rgef-1) promoters to assess localization. Using these promoters, we found that PNG-1 was consistently localized to the cytoplasm (Fig. 5G,H). This is in agreement with the cytoplasmic localization of PNGases in yeast, mammalian, and plant cells (Suzuki et al., 2001; Hirsch et al., 2003; Diepold et al., 2007).

png-1 acts from epithelia and neurons to regulate branching The broad expression of png- 1 during L4 when VC neurons extend axons and branch did not immediately suggest a cellular site of action. We therefore used cell-specific promoters to drive PNG-1 in either neurons or epithelial cells to assess rescue of the png-1(cy9) axon branching and outgrowth defects. Heterologous promoters were used to drive $p n g-1$ expression in VCs (cat- 1 and unc-4), all neurons (rgef-1), all epithelia (col-10 and ajm-1), and vulval ( $s q v-1$ and $s q v-3)$ or subsets of vulval or uterine epithelial cells (egl-17 and ocr-4) (supplemental Table 2, available at www. jneurosci.org as supplemental material). We found that the penetrance of the png-1(cy9) VC arbor overgrowth phenotype ( $\sim 64 \%$ ) was reduced when $p n g-1$ was expressed from all epithelial cells (29-36\%), neuronal cells $(\sim 40 \%)$, and vulval cells (32$37 \%$ ) but not from uterine cells ( $\sim 70 \%)$ or a subset of vulval cells (61-67\%) (Fig. 6A). Similar positive results with pan-epithelial 
A PNG-1

$\Delta \mathrm{C}$-term
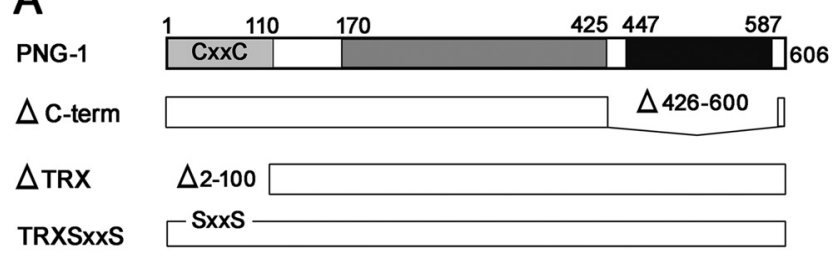

B

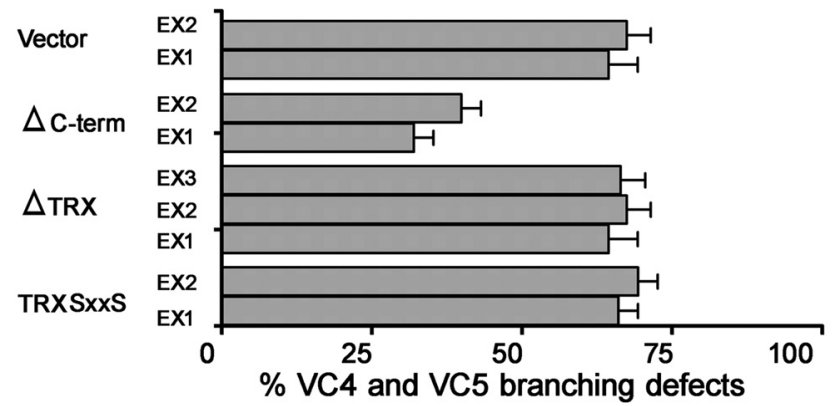

Figure 7. The C-terminal domain is not essential for PNG-1 axon branching functions. $A$, PNG-1 structure-function analysis was performed by generating in-frame deletions of the thioredoxin and C-terminal domains and site-directed mutagenesis of the conserved Cys catalytic residues in the thioredoxin domain and scoring for rescue of VC4-5 branching defects in png-1(cy9); cyls4[Pcat-1::GFP] animals after germ-line transformation. B, Multiple extrachromosomal (Ex) lines were generated for each construct. VC4-5 branching defects were rescued with full-length and C-terminal deleted PNG-1 constructs but not thioredoxin mutants. Constructs were tagged with GFP to verify expression. Error bars denoteSEM; $n=48-120$ animals.

and pan-neuronal promoters were obtained for rescue of the DVB overextension and branching defects (Fig. 6B,C). These findings are consistent with png-1 acting in a non-cell-autonomous manner from multiple tissues to limit neuronal outgrowth and branching. PNG-1 activity in either vulval cells or VC neurons appears to be sufficient to prevent VC branch overgrowth. However, these findings do not exclude the possibility that some functions of $p n g-1$ on axon outgrowth and branching also take place cell autonomously. Interestingly, png-1 overexpression in wild-type animals (data not shown) never appeared to cause a decrease in VC branching, suggesting that png-1 is not a ratelimiting step in regulating VC axon branching.

\section{The thioredoxin and PNGase domains but not the $\mathrm{C}$ terminus} are important for axon branching

The highly penetrant axon branching defects associated with the missense mutation in $p n g-1(c y 8)$, which disrupts the active site Cys (C251Y), is strong evidence that the PNGase/TGase core domain is functionally important. To determine whether the thioredoxin and the predicted C-terminal mannose binding domain are also important for PNG-1 axon branching functions, we generated PNG-1::GFP mutants with specific domain deletions or mutations and assessed their ability to rescue $p n g-1^{-/-}$branching defects (Fig. 7). We found that truncated PNG-1 protein missing the C-terminal mannose binding domain (residues 426-600) was capable of restoring normal VC axon branching when expressed in png-1(cy9) animals. Transgenic lines carrying the C-terminal deleted PNG-1 mutant displayed 32-39\% excess VC branching defects compared with control animals (65-68\%) and similar to the rescue obtained with full-length PNG-1 (21-25\%). Conversely, expression of PNG-1 lacking the thioredoxin domain (residues 2-100) (65-67\%) or carrying a site-specific disruption of the CGPC (mutated to SGPS) redox active site (66-69\%) did not rescue png-1(cy9) branching defects. Western blots using anti-
GFP antibodies were performed to confirm protein expression (data not shown). These results indicate that the predicted C-terminal mannose binding domain is dispensable for PNG-1 axon branching functions.

\section{png-1-like branching defects are not displayed in mutants that disrupt ER homeostasis}

PNGases act on misfolded glycoprotein substrates targeted for degradation by ER quality control pathways. We therefore wanted to examine the possibility that excess axon branching was a non-specific effect of misregulated cellular processes caused by the intracellular accumulation of misfolded glycoproteins. To address this, we crossed our GFP reporter lines into mutants that disrupt highly conserved components of the unfolded protein response (UPR) and ERAD adaptive response pathways to ER stress and examined VC4-5, AVL, and DVB axon morphology. The worm homologs of several core pathway components, such as Ire1 (Shen et al., 2001), Pek1/Perk (Shen et al., 2001), and Hrd1/Der3 (Sasagawa et al., 2007), have been shown recently to function in the cellular responses to ER stress in C. elegans. Inactivation of these genes by mutation or RNA interference depletion trigger the widespread expression of a UPR inducible Phsp-4::GFP reporter indicative of elevated levels of ER stress throughout the animal (Calfon et al., 2002; Sasagawa et al., 2007). Our analysis failed to detect obvious VC, AVL, or DVB axon branching abnormalities in ire-1(ok799), pek-1(ok275), and hrd1(tm1743) deletion mutants, arguing against nonspecific effects caused by dysregulation of ER homeostasis (supplemental Table 3, available at www.jneurosci.org as supplemental material).

\section{png-1 and rad-23 mutants display similar axon branching defects}

Yeast and mammalian PNGases interact with the proteasome through a physical association with Rad-23, a highly conserved protein known for its role in DNA repair that contains an $\mathrm{N}$-terminal ubiquitin-like (UBQ) domain, two ubiquitin-associated (UBA) domains, and a xeroderma pigmentosum complementation group C binding (XPC) domain (Suzuki et al., 2001; Katiyar et al., 2004; Lee et al., 2005). A genome-wide BLAST (basic local alignment search tool) search identified ZK20.3/rad-23 (GenBank no. CAA93780) as the only Rad-23 homolog in C. elegans (36\% identity to human RAD-23A; GenBank accession number NM005053.2). We therefore examined the rad-23(tm2595) deletion mutant (a gift from S. Mitani, Department of Physiology, Tokyo Women's Medical University School of Medicine, Tokyo, Japan) for png-1like neuronal defects (Fig. 8A). VC4-5 morphology appeared normal in rad-23(tm2595), but we did observe that $6 \%(n=137)$ of AVL axons displayed inappropriate branches near the vulva (Fig. $8 B-D), 40 \%(n=150)$ of DVB axons displayed an overextension defect (Fig. $8 E, I)$, and $6 \%(n=98)$ of the overextended DVB axons displayed axon branches at the vulva (Fig. $8 F, J)$ that were similar to those displayed by png-1 mutants. Like png-1, branching defects in rad-23 mutants did not occur at the L3 stage before vulval organogenesis.

The finding that png- 1 and rad-23 mutants share similar AVL and DVB phenotypes raised the question as to whether they also act in the same genetic pathway. We therefore constructed png-1; rad-23 double mutants and examined them for genetic interactions. The penetrance of AVL branching defects did not significantly differ in the png-1(cy9); rad-23(tm2595) double (23\%; $n=149$ ) (Fig. $8 C, D)$ compared with the png-1(cy9) single (25\%) mutant. Similarly, the penetrance of DVB defects in the png-1(cy9); rad23(tm2595) double (40\%, $n=150$ extension and $12 \%, n=83$ 
A
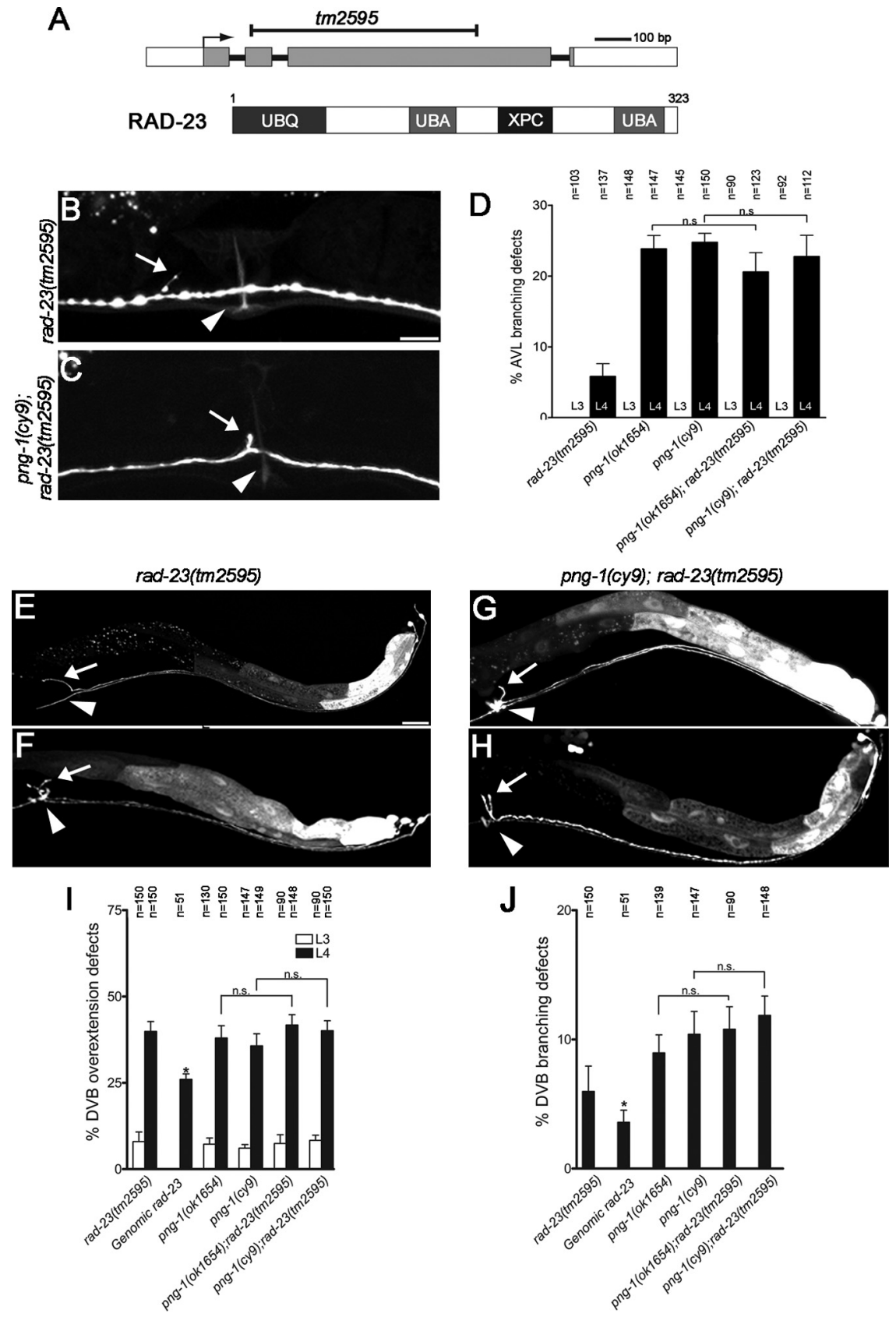

$\mathbf{J}$

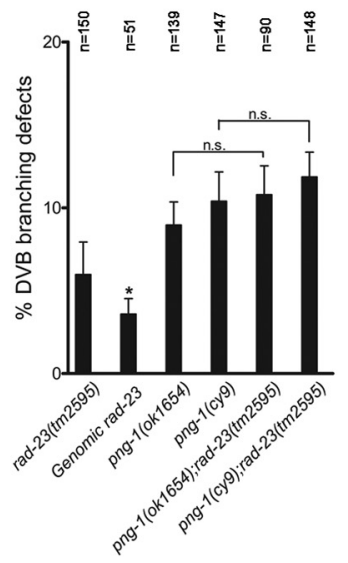

Figure 8. png-1 and rad-23 act in a common genetic pathway to limit axon branching at the vulva. $A$, Genomic and protein structure of RAD-23 showing position and size of the tm2595 deletion and position of conserved UBL, UBA, and XPC domains. B, C, AVL axons, visualized in an Punc-25::GFP; unc-30(e191) background, displaying inappropriate branching (arrows) at the vulva (arrowheads) in rad23(tm2595) (B) and png-1(cy9); rad-23(tm2595) (C) adult worms. D. The penetrance of vulval branches in L4 animals do not significantly differ in png-1 single and png-1; rad-23 double mutants, indicating that these genes act in the same genetic pathway to prevent ectopic AVL branching. $\boldsymbol{E}-\boldsymbol{H}$, DVB axons, visualized using an Pflp-10::GFP reporter, displaying overextension defects (arrows) in rad-23(tm2595) (E) and png-1(cy9); rad-23(tm2595) (G,I) at the vulva (arrowheads) in adult worms. A subset of overextended DVB axons also display inappropriate branching (arrows) at the vulva (arrowhead) $(\boldsymbol{F}, \boldsymbol{H})$. The penetrance of overextension $(\boldsymbol{I})$ or branching $(J)$ defects in $\mathrm{L} 4$ animals do not significantly differ in png-1 single and png-1; rad-23 double mutants, indicating these genes act in the same genetic pathway to prevent DVB overextension and ectopic branching. AVL and DVB defects are not observed $(\boldsymbol{D})$ or are less severe $(\boldsymbol{I})$ when counts are performed before vulval organogenesis at $L 3$. Scale bars: $\boldsymbol{B}, \boldsymbol{C}, 10 \mu \mathrm{m} ; \boldsymbol{E}-\boldsymbol{H}, 20 \mu \mathrm{m}$. Error bars for rad-23 genomic rescue denote SE of proportion. ${ }^{*} p<0.05 ; n=51$. Error bars for all other data denote SEM. $\chi^{2}$ analysis was used to determine that double mutants do not significantly differ (ns) from single mutants.

branching) (Fig. 8G-J) and the png-1(cy9) (36\% extension and $10 \%$ branching) and rad-23(tm2595) (40\% extension) single mutants were not significantly different. Similar results were observed in double mutants with png-1(ok1654). These results indicate that rad-23 and png- 1 act in the same genetic pathway to prevent AVL and DVB neurons from branching or overextending at the vulva.

\section{Discussion}

Because the molecular identification of cytoplasmic PNGase as the gene disrupted in an $S$. cerevisiae mutant that lacked $N$-glycanase activity, highly conserved orthologs have been identified in the genomes of fungi, plants, and animals (Suzuki et al., 2000). Several in vitro studies using human cell lines point to a role for PNGases in the proteasomal-dependent turnover or processing of glycoproteins or glycopeptides that originate in the ER and are retrotranslocated to the cytosol. These roles include the catabolism of misfolded or aberrant glycoproteins during ERAD (Hirsch et al., 2003; Blom et al., 2004; Kim et al., 2006) and the cytoplasmic processing of glycosylated peptide fragments to generate MHC class I epitopes (Selby et al., 1999; Altrich-VanLith et al., 2006; Kario et al., 2008). However, the developmental and physiological consequences of PNGase deficiency in animal models await the phenotypic analysis of knock-out mutants. Here we describe for the first time mutations in the sole C. elegans PNGase revealing a previously unknown role for PNGase and its binding partner Rad-23 in regulating neuronal branching during morphogenesis of an organ system.

The most prominent defect in png-1 animals is the overgrowth and increased branching of the VC terminal arbor, the site of synapses to the HSN motor neurons and the vulval muscles (Feinberg et al., 2008). Current models of egg laying suggest that acetylcholine release from VC neurons acts to both promote vulval muscle contraction directly while simultaneously inhibiting the stimulatory effect of HSN activity on vulval muscles (Waggoner et al., 1998; Bany et al., 2003). Evidence for an inhibitory role comes from the hyperactive egg-laying phenotype displayed by animals in which VC neurons have been genetically or laser ablated (Bany et al., 2003). In contrast, we find that png-1 animals display a mild egg-retention phenotype, suggesting that excess branching may contribute to a strengthening of VC inhibition on HSN activity. The opposite phenotype is observed in png- 1 ; egl-1 (gf) animals that lack HSN neurons, consistent with overstimulation of vulval muscles leading to increased egg laying. PNG-1 activity therefore appears important to limit the extent of VC arborization to prevent overstimulation of the egg-laying circuit.

The vulva, a source of innervation and axon branching cues (Li and Chalfie, 1990; Garriga et al., 1993; Shen et al., 2004; Adler 
et al., 2006), is formed during the L3 and L4 stages in a cellular environment occupied by preexisting axon tracts. A striking feature of png-1 and rad-23 mutants is that axon branching defects are only found in proximity to the vulva. The observation that AVL and DVB neurons sprout vulval axon branches de novo in our mutants beginning at L4 from axons that were unbranched at the beginning of vulval organogenesis clearly indicates that normally quiescent axons are intrinsically able to respond to these cues but are normally prevented from doing so. This suggests that PNG-1 and RAD-23 may be part of an organ-specific mechanism to limit responses to late-acting peripheral innervation cues to neurons involved in egg laying such as the VC motor neurons and not to axonal tracts that are present before vulval organogenesis. This notion may be similar to mechanisms known to limit or alter neuronal responses to hierarchically acting pathfinding cues during wiring of the CNS (Stein and Tessier-Lavigne, 2001).

PNGases are cytoplasmic de- $N$-glycosylation enzymes. The domain structure of PNG-1 consists of an N-terminal thioredoxin, a central PNGase, and a predicted C-terminal mannose binding domain. The thioreductase and $\mathrm{N}$-glycanase activities of recombinant $C$. elegans PNG-1 have been verified previously (Kato et al., 2007; Suzuki et al., 2007). The disruption of the catalytic Cys (C251Y) in cy8 is clear evidence that $N$-glycanase activity is important to mediate axon branching functions. In vitro enzymatic assays using C251Y PNG-1 protein confirm that this mutation specifically abolishes deglycosylation activity without disrupting the thioreductase activity (Suzuki et al., 2007). In addition, the similar phenotypic penetrances of the putative $c y 9$ null and the ok1654 deletion allele suggests that the "glycanasedead" cy 8 mutation is the equivalent of a complete loss of protein function. The requirement for the thioredoxin domain to rescue branching defects may be related to studies that show a reduced redox state is necessary for optimal PNGase activity (Suzuki et al., 2007).

This study was limited to PNG-1 function in the nervous system and did not examine potential roles in glycoprotein catabolism. However, it is reasonable to ask whether png-1 acts via an ERAD-like pathway to regulate branching. PNG-1 differs structurally from other animal PNGases, such as those of flies and mammals, in that it contains a thioredoxin instead of a PUB domain at its $\mathrm{N}$ terminus (Suzuki et al., 2007). The PUB domain in mouse $N$-glycanase is the site of interactions with several known ERAD components, including the AAA ATPase p97, the ubiquitin protein ligase AMFR, and Derlin1 (Katiyar et al., 2005; $\mathrm{Li}$ et al., 2005, 2006). The absence of this domain in PNG-1 may suggest that axon branching functions may be primarily independent of possible PNG-1 roles in maintaining ER homeostasis. Similarly, the predicted mannose binding C-terminal domain, which in mouse PNGase has been shown to influence the rate of deglycosylation of an ERAD substrate in vitro (Zhou et al., 2006), is dispensable for axon branching, suggesting other roles to account for its conservation in PNG-1. The absence of more widespread neuronal defects outside of the vulval region in $p n g-1$ mutants as might be expected from loss of a general ER quality control component and the lack of branching phenotypes in mutants with elevated levels of ER stress are also consistent with an ERAD-independent role for PNG-1 in axon branching.

The finding that PNG-1 can act non-cell autonomously from vulval epithelia to regulate neuronal branching has important mechanistic implications and suggests for the first time that PNGases also participate in intercellular signaling. A cell extrinsic effect can be explained if PNGase activity in epithelial cells influences the secretion or cell-surface expression of an axon branch-

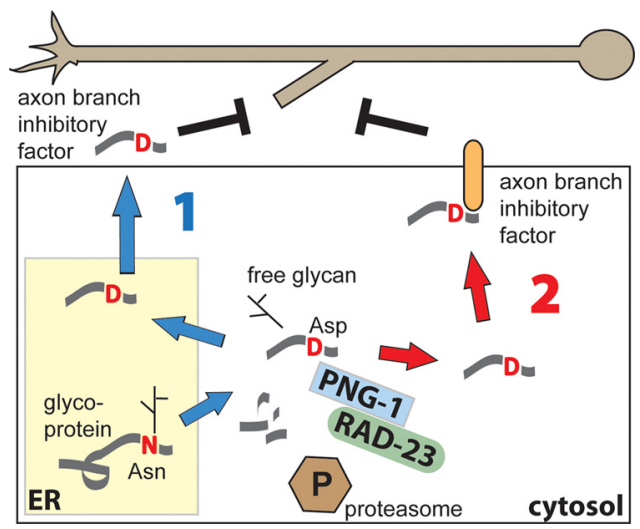

Figure 9. Model for cytosolic PNGase activity acting non-cell autonomously to limit axon branching. Our findings suggest that epithelial cells provide a branch inhibitory factor to nearby axons (or limit a branch promoting factor) to prevent ectopic axon branching or limit the size of axon arbors. We propose that this factor is derived from a specific glycoprotein or glycopeptide substrate that is retrotranslocated from the ER to the cytoplasm and processed into a bioactive peptide by a combination of $\mathrm{N}$-glycanase activity and proteolytic degradation. PNGasemediated posttranslational modifications include the release of a glycan and conversion of Asn to Asp. The resulting "activated" peptide could then act directly by reentering the secretory pathway (1) or indirectly by interacting with cell-surface receptors (2) to inhibit axon branching. A PNG-1-RAD-23 complex is hypothesized to be involved in some contexts.

ing molecule such that png-1-deficient cells would present either too much of a branch promoting or too little of a branch inhibitory factor, resulting in a hyperinnervation phenotype.

At the same time, we cannot entirely exclude a cellautonomous role given that png-1 defects were also rescued with neuronal-specific expression. However, a cell extrinsic role is not incompatible with a cellular focus in VC neurons. First, because VC4 and VC5 arbors overlap, each may mutually limit the branching of the other through a lateral inhibitory mechanism akin to that involved in dendritic tiling (Grueber et al., 2002). Alternatively, a factor expressed from VC neurons may act in an autocrine manner to inhibit axon overgrowth and branching. A classic example of autocrine signaling is the action of hepatocytic growth factor on the elongation of sympathetic axons (Yang et al., 1998).

To explain the non-cell-autonomous effect, we propose that PNG-1 regulates branching in a process that involves dislocation of a specific $N$-glycoprotein or glycopeptide from the ER lumen in which $N$-glycosylation takes place to the cytosol where deglycosylation generates a bioactive form that can then interact directly or indirectly with cell-surface or secreted factors (Fig. 9). It is possible that, in this context, PNG-1 acts as a specific posttranslational modification enzyme to alter protein function through the removal a specific oligosaccharide or the addition of a negatively charged amino acid (Asn to Asp).

Any model must also include Rad-23, which forms a complex with PNGase and shown by us to interact genetically to prevent AVL and DVB axon branching. Rad-23 proteins interact with the proteasome and multiubiquitin chains through their N-terminal ubiquitin-like and two ubiquitin-associated domains (Madura, 2004), suggesting a role for the ubiquitin-proteasome pathway in PNG-1-mediated axon branching. This is consistent with the fact that proteasomal degradation in the cytosol is an important feature of all known PNGase-associated pathways, such as ERADlinked glycoprotein catabolism and MHC peptide processing. We note that Rad-23 proteins have been shown to act in both escort-like roles to shuttle substrates targeted for degradation to the proteasome (Madura, 2004) as well as antiproteolytic roles to 
stabilize proteins by blocking proteasome interaction ( $\mathrm{Ng}$ et al., 2003). Distinguishing between these possibilities and gaining additional insight into the PNG-1 axon branching pathway will require identification of the molecular target of PNGase activity.

It is interesting to speculate of a link between the role mammalian PNGases play in the deglycosylation of peptide epitopes required for proper MHC class I molecule presentation and the newly emerging roles for MHC class I molecules in axon pathfinding and synaptic remodeling during development (Corriveau et al., 1998; Huh et al., 2000). In mammals, antigenic peptides are translocated from the cytosol into the ER via the transporter associated with antigen processing (TAP), a member of the ATP binding cassette $(A B C)$ transporter family. Although $C$. elegans, like other invertebrates, do not possess MHC molecules or other components of an adaptive immune system, they do possess 60 members of the ABC transporter family, including several TAPlike genes (Zhao et al., 2007), suggesting the possibility that a cytosolic peptide or proteolytic fragment may reenter the ER and be processed through the secretory pathway (Fig. 9), although such a pathway has not been demonstrated. Nevertheless, given our results, it will be important to determine whether mammalian PNGases contribute to MHC class I signaling in the nervous system.

In summary, our findings are consistent with png- 1 acting in a pathway to regulate cues that limit the extent of axon branching during organ innervation in C. elegans. Our findings suggest that animal PNGases should be examined for roles in neural wiring and plasticity in addition to their protective "housekeeping" roles in cellular homeostasis.

\section{References}

Adler CE, Fetter RD, Bargmann CI (2006) UNC-6/Netrin induces neuronal asymmetry and defines the site of axon formation. Nat Neurosci 9:511-518.

Altrich-VanLith ML, Ostankovitch M, Polefrone JM, Mosse CA, Shabanowitz J, Hunt DF, Engelhard VH (2006) Processing of a class I-restricted epitope from tyrosinase requires peptide $\mathrm{N}$-glycanase and the cooperative action of endoplasmic reticulum aminopeptidase 1 and cytosolic proteases. J Immunol 177:5440-5450.

Altun-Gultekin Z, Andachi Y, Tsalik EL, Pilgrim D, Kohara Y, Hobert O (2001) A regulatory cascade of three homeobox genes, ceh-10, ttx-3 and ceh-23, controls cell fate specification of a defined interneuron class in $C$. elegans. Development 128:1951-1969.

Bany IA, Dong MQ, Koelle MR (2003) Genetic and cellular basis for acetylcholine inhibition of Caenorhabditis elegans egg-laying behavior. J Neurosci 23:8060-8069.

Blom D, Hirsch C, Stern P, Tortorella D, Ploegh HL (2004) A glycosylated type I membrane protein becomes cytosolic when peptide: $\mathrm{N}$-glycanase is compromised. EMBO J 23:650-658.

Calfon M, Zeng H, Urano F, Till JH, Hubbard SR, Harding HP, Clark SG, Ron D (2002) IRE1 couples endoplasmic reticulum load to secretory capacity by processing the XBP-1 mRNA. Nature 415:92-96.

Colavita A, Tessier-Lavigne M (2003) A Neurexin-related protein, BAM-2, terminates axonal branches in C. elegans. Science 302:293-296.

Corriveau RA, Huh GS, Shatz CJ (1998) Regulation of class I MHC gene expression in the developing and mature CNS by neural activity. Neuron 21:505-520.

Cui M, Han M (2003) Cis regulatory requirements for vulval cell-specific expression of the Caenorhabditis elegans fibroblast growth factor gene egl-17. Dev Biol 257:104-116.

Diepold A, Li G, Lennarz WJ, Nürnberger T, Brunner F (2007) The Arabidopsis AtPNG1 gene encodes a peptide: N-glycanase. Plant J 52:94-104.

Eastman C, Horvitz HR, Jin Y (1999) Coordinated transcriptional regulation of the unc-25 glutamic acid decarboxylase and the unc-47 GABA vesicular transporter by the Caenorhabditis elegans UNC-30 homeodomain protein. J Neurosci 19:6225-6234.

Feinberg EH, Vanhoven MK, Bendesky A, Wang G, Fetter RD, Shen K, Bargmann CI (2008) GFP reconstitution across synaptic partners
(GRASP) defines cell contacts and synapses in living nervous systems. Neuron 57:353-363.

Garriga G, Desai C, Horvitz HR (1993) Cell interactions control the direction of outgrowth, branching and fasciculation of the HSN axons of Caenorhabditis elegans. Development 117:1071-1087.

Glebova NO, Ginty DD (2005) Growth and survival signals controlling sympathetic nervous system development. Annu Rev Neurosci 28:191222

Grueber WB, Jan LY, Jan YN (2002) Tiling of the Drosophila epidermis by multidendritic sensory neurons. Development 129:2867-2878.

Hirsch C, Blom D, Ploegh HL (2003) A role for N-glycanase in the cytosolic turnover of glycoproteins. EMBO J 22:1036-1046.

Huh GS, Boulanger LM, Du H, Riquelme PA, Brotz TM, Shatz CJ (2000) Functional requirement for class I MHC in CNS development and plasticity. Science 290:2155-2159.

Hwang HY, Horvitz HR (2002) The SQV-1 UDP-glucuronic acid decarboxylase and the SQV-7 nucleotide-sugar transporter may act in the Golgi apparatus to affect Caenorhabditis elegans vulval morphogenesis and embryonic development. Proc Natl Acad Sci U S A 99:14218-14223.

Jose AM, Bany IA, Chase DL, Koelle MR (2007) A specific subset of TRPV channel subunits in Caenorhabditis elegans endocrine cells function as mixed heteromers to promote neurotransmitter release. Genetics 175:93-105.

Kario E, Tirosh B, Ploegh HL, Navon A (2008) N-linked glycosylation does not impair proteasomal degradation but affects class I major histocompatibility complex presentation. J Biol Chem 283:244-254.

Katiyar S, Li G, Lennarz WJ (2004) A complex between peptide:N-glycanase and two proteasome-linked proteins suggests a mechanism for the degradation of misfolded glycoproteins. Proc Natl Acad Sci U S A 101:13774-13779.

Katiyar S, Joshi S, Lennarz WJ (2005) The retrotranslocation protein Derlin-1 binds peptide:N-glycanase to the endoplasmic reticulum. Mol Biol Cell 16:4584-4594.

Kato T, Kawahara A, Ashida H, Yamamoto K (2007) Unique peptide:Nglycanase of Caenorhabditis elegans has activity of protein disulphide reductase as well as of deglycosylation. J Biochem 142:175-181.

Kim I, Ahn J, Liu C, Tanabe K, Apodaca J, Suzuki T, Rao H (2006) The Png1-Rad23 complex regulates glycoprotein turnover. J Cell Biol 172:211-219.

Kitajima K, Suzuki T, Kouchi Z, Inoue S, Inoue Y (1995) Identification and distribution of peptide:N-glycanase (PNGase) in mouse organs. Arch Biochem Biophys 319:393-401.

Köppen M, Simske JS, Sims PA, Firestein BL, Hall DH, Radice AD, Rongo C, Hardin JD (2001) Cooperative regulation of AJM-1 controls junctional integrity in Caenorhabditis elegans epithelia. Nat Cell Biol 3:983-991.

Lee JH, Choi JM, Lee C, Yi KJ, Cho Y (2005) Structure of a peptide:Nglycanase-Rad23 complex: insight into the deglycosylation for denatured glycoproteins. Proc Natl Acad Sci U S A 102:9144-9149.

Li C, Chalfie M (1990) Organogenesis in C. elegans: positioning of neurons and muscles in the egg-laying system. Neuron 4:681-695.

Li G, Zhou X, Zhao G, Schindelin H, Lennarz WJ (2005) Multiple modes of interaction of the deglycosylation enzyme, mouse peptide N-glycanase, with the proteasome. Proc Natl Acad Sci U S A 102:15809-15814.

Li G, Zhao G, Zhou X, Schindelin H, Lennarz WJ (2006) The AAA ATPase p97 links peptide $\mathrm{N}$-glycanase to the endoplasmic reticulum-associated E3 ligase autocrine motility factor receptor. Proc Natl Acad Sci U S A 103:8348-8353.

Lickteig KM, Duerr JS, Frisby DL, Hall DH, Rand JB, Miller DM 3rd (2001) Regulation of neurotransmitter vesicles by the homeodomain protein UNC-4 and its transcriptional corepressor UNC-37/groucho in Caenorhabditis elegans cholinergic motor neurons. J Neurosci 21:2001-2014.

Madura K (2004) Rad23 and Rpn10: perennial wallflowers join the melee. Trends Biochem Sci 29:637-640.

McGovern M, Yu L, Kosinski M, Greenstein D, Savage-Dunn C (2007) A role for sperm in regulation of egg-laying in the nematode C. elegans. BMC Dev Biol 7:41.

Mehta N, Loria PM, Hobert O (2004) A genetic screen for neurite outgrowth mutants in Caenorhabditis elegans reveals a new function for the F-box ubiquitin ligase component LIN-23. Genetics 166:1253-1267.

Ng JM, Vermeulen W, van der Horst GT, Bergink S, Sugasawa K, Vrieling H, Hoeijmakers JH (2003) A novel regulation mechanism of DNA repair 
by damage-induced and RAD23-dependent stabilization of xeroderma pigmentosum group C protein. Genes Dev 17:1630-1645.

Peckol EL, Zallen JA, Yarrow JC, Bargmann CI (1999) Sensory activity affects sensory axon development in C. elegans. Development 126:1891-1902.

Sasagawa Y, Yamanaka K, Ogura T (2007) ER E3 ubiquitin ligase HRD-1 and its specific partner chaperone BiP play important roles in ERAD and developmental growth in Caenorhabditis elegans. Genes Cells 12:10631073.

Seiler S, Plamann M (2003) The genetic basis of cellular morphogenesis in the filamentous fungus Neurospora crassa. Mol Biol Cell 14:4352-4364.

Selby M, Erickson A, Dong C, Cooper S, Parham P, Houghton M, Walker CM (1999) Hepatitis C virus envelope glycoprotein E1 originates in the endoplasmic reticulum and requires cytoplasmic processing for presentation by class I MHC molecules. J Immunol 162:669-676.

Shen K, Fetter RD, Bargmann CI (2004) Synaptic specificity is generated by the synaptic guidepost protein SYG-2 and its receptor, SYG-1. Cell 116:869-881.

Shen X, Ellis RE, Lee K, Liu CY, Yang K, Solomon A, Yoshida H, Morimoto R, Kurnit DM, Mori K, Kaufman RJ (2001) Complementary signaling pathways regulate the unfolded protein response and are required for $C$. elegans development. Cell 107:893-903.

Stein E, Tessier-Lavigne M (2001) Hierarchical organization of guidance receptors: silencing of netrin attraction by slit through a Robo/DCC receptor complex. Science 291:1928-1938.
Suzuki T (2007) Cytoplasmic peptide:N-glycanase and catabolic pathway for free N-glycans in the cytosol. Semin Cell Dev Biol 18:762-769.

Suzuki T, Park H, Hollingsworth NM, Sternglanz R, Lennarz WJ (2000) PNG1, a yeast gene encoding a highly conserved peptide:N-glycanase. J Cell Biol 149:1039-1052.

Suzuki T, Park H, Kwofie MA, Lennarz WJ (2001) Rad23 provides a link between the Png1 deglycosylating enzyme and the $26 \mathrm{~S}$ proteasome in yeast. J Biol Chem 276:21601-21607.

Suzuki T, Tanabe K, Hara I, Taniguchi N, Colavita A (2007) Dual enzymatic properties of the cytoplasmic peptide: $\mathrm{N}$-glycanase in C. elegans. Biochem Biophys Res Commun 358:837-841.

Waggoner LE, Zhou GT, Schafer RW, Schafer WR (1998) Control of alternative behavioral states by serotonin in Caenorhabditis elegans. Neuron 21:203-214.

Yang XM, Toma JG, Bamji SX, Belliveau DJ, Kohn J, Park M, Miller FD (1998) Autocrine hepatocyte growth factor provides a local mechanism for promoting axonal growth. J Neurosci 18:8369-8381.

Zhao Z, Thomas JH, Chen N, Sheps JA, Baillie DL (2007) Comparative genomics and adaptive selection of the ATP-binding-cassette gene family in Caenorhabditis species. Genetics 175:1407-1418.

Zhou X, Zhao G, Truglio JJ, Wang L, Li G, Lennarz WJ, Schindelin H (2006) Structural and biochemical studies of the C-terminal domain of mouse peptide-N-glycanase identify it as a mannose-binding module. Proc Natl Acad Sci U S A 103:17214-17219. 